\title{
Magnetoresistance of Mn-decorated topological line defects in graphene
}

\author{
J. T. Obodo, M. Upadhyay Kahaly, and U. Schwingenschlögl* \\ PSE Division, KAUST, Thuwal 23955-6900, Kingdom of Saudi Arabia
}

(Received 25 September 2014; published 13 January 2015)

\begin{abstract}
We study the spin polarized transport through Mn-decorated 8-5-5-8 topological line defects in graphene using the nonequilibrium Green's function formalism. Strong preferential bonding overcomes the high mobility of transition metal atoms on graphene and results in stable structures. Despite a large distance between the magnetic centers, we find a high magnetoresistance and attribute this unexpected property to very strong induced $\pi$ magnetism, in particular for full coverage of all octagonal hollow sites by $\mathrm{Mn}$ atoms. In contrast to the magnetoresistance of graphene nanoribbon edges, the proposed system is well controlled and therefore suitable for applications.
\end{abstract}

DOI: 10.1103/PhysRevB.91.014413

\section{INTRODUCTION}

Carbon nanomaterials [1], such as graphene, fullerenes [2], and nanotubes [3], host exciting physical and chemical phenomena and therefore are provoking a lot of scientific activity. The spatial confinement of the electronic states in such systems is key for their properties and also determines the interaction with other matter. In its ideal form, graphene consists of a two-dimensional honeycomb lattice of $s p^{2}$ hybridized $\mathrm{C}$ atoms. However, various structural imperfections can arise, for example line defects, for which the formation of pairs of pentagonal and octagonal rings allows the system to reduce the internal strain. This defect with fractional chirality was predicted previously for nanotubes [4] and, referring to the arrangement of the rings, is called 8-5-5-8 topological line defect. It has been observed in as-grown graphene [5] and can also be induced in a controlled fashion by electron irradiation $[6,7]$. In general, the intentional introduction of defects into graphene is a topic that currently receives enormous attention, because it can lead to a multitude of applications [8-14]. Moreover, the interactions between defects in graphene and metal atoms have been explored extensively in the literature [15-19]. Selective deposition of $\mathrm{Pt}$ atoms at graphene line defects recently has been demonstrated in Ref. [20].

Magnetic sensors based on the magnetoresistance of a material have been investigated intensively for quite a while [21,22]. In addition, magnetoresistant materials are of great interest in the field of spintronics, see Ref. [23] and the references therein. In this context, graphene nanoribbons recently were found to offer high magnetoresistance [24], although rough edges lead to enhanced scattering and little control over the material properties. Moreover, the growth of nanoribbons on a substrate always introduces some amount of disorder [25]. On the other hand, adsorption of transition-metal atoms can be utilized to introduce spin polarization in graphene [26]. In addition, at least for a low concentration of adatoms, the magnetization can be tuned by varying the Fermi energy, either by applying a gate voltage or by suitable doping [27]. Since adsorption and desorption on surfaces are largely controlled by surface defects [28], stable line defects in graphene are expected to trigger a selective decoration, which is useful for engineering spin transport channels. The one-dimensional

*Udo.Schwingenschlogl@kaust.edu.sa
PACS number(s): 72.80.Vp, 73.43.Qt, 81.05.ue

transport through such an arrangement resembles that through an atomic wire. However, the distance between the magnetic centers in decorated line defects is typically much too large to induce magnetoresistance.

In the present work, we propose a strategy to overcome the limited controllability that graphene nanoribbon edges suffer from. For introducing spin polarization and magnetoresistance in graphene, we decorate a 8-5-5-8 topological line defect with $\mathrm{Mn}$ atoms. Strong preferential bonding results in very stable structures. Despite the large distance between the magnetic centers, we demonstrate a high magnetoresistance. This effect is explained by a very strong induced $\pi$ magnetism of the graphene host, which results in directed spin transport.

\section{TECHNIQUES}

Our calculations are based on density functional theory and the generalized gradient approximation to the exchange correlation potential in the Ceperley-Alder flavor. We use the SIESTA code [29], which employs linear combinations of atomic orbitals as basis functions (double $\zeta$ polarization) and normconserving pseudopotentials. An energy cutoff of $300 \mathrm{Ry}$ is used. The pseudopotentials and basis sets have been tested for diamond as well as graphite. Moreover, the Brillouin zone is sampled on a $30 \times 1 \times 30$ Monkhorst-Pack $k$ mesh. Geometry optimizations are carried out by the conjugate gradient method until the forces acting on the atoms have declined below $0.04 \mathrm{eV} / \AA$. Band structures are studied along the high symmetry direction $\Gamma-\mathrm{K}$ and along the line defect $(\Gamma-\mathrm{C})$. The transport is determined by the nonequilibrium Green's function approach as implemented in the SMEAGOL package [30], where the scattering region consists of eight replicas of the cell shown in Fig. 1(a), stacked along the $z$ direction, and each lead of two such replicas. The cell of defective graphene is shown in Fig. 1(b). Because Mn decoration is favorable on the line defect irrespective of the Mn coverage [31], we address only the cases of 50\% and $100 \%$ occupation, see Fig. 1(c). Periodic boundary conditions are imposed to the leads, making them semi-infinite, and the electronic temperature is set to $300 \mathrm{~K}$. The technical parameters are the same as in the electronic structure calculations, except that a $5 \times 1 \times 1 k$ mesh is used. We do not take into account the spin orbit interaction, since the effects are expected to be negligible. 


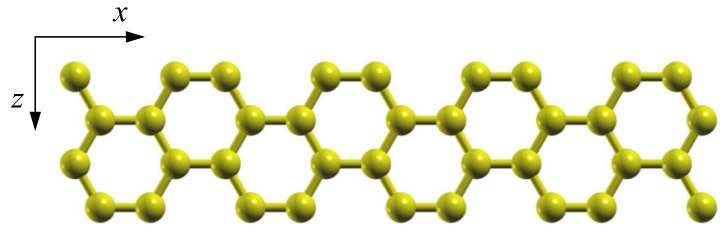

(a)

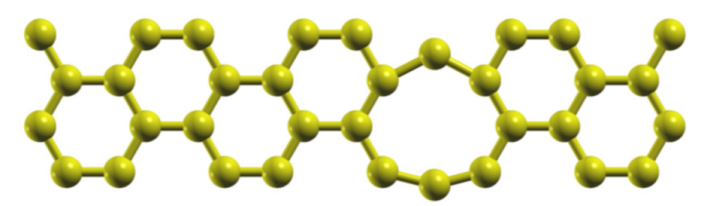

(b)

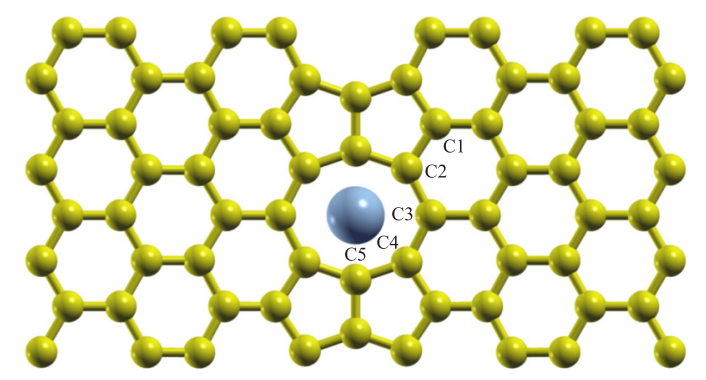

(c)

FIG. 1. (Color online) Structure of (a) graphene, (b) graphene with line defect, and (c) graphene with 50\% Mn-decorated line defect. The labeling of the $\mathrm{C}$ atoms takes into account the symmetry of the structure.

The spin-dependent current $I_{\alpha}(V)$ is obtained from the Landauer-Büttiker formula,

$$
I_{\alpha}(V)=\frac{e}{h} \int_{-\infty}^{\infty} T_{\alpha}(E, V)\left[f_{L}\left(E-\mu_{L}\right)-f_{R}\left(E-\mu_{R}\right)\right] d E,
$$

where $\mu_{L / R}$ is the chemical potential of the left/right electrode, $V$ the applied bias voltage, $f_{L / R}$ the Fermi-Dirac distribution function, $T_{\alpha}$ the transmission, and $\alpha$ the spin. The spindependent zero bias conductance is

$$
G_{\alpha}(E)=\frac{e^{2}}{h} T_{\alpha}(E)=\frac{e^{2}}{h} \operatorname{Tr}\left[\Gamma_{L} G^{R} \Gamma_{R} G^{A}\right]_{\alpha},
$$

where $\Gamma_{L} / \Gamma_{R}$ is the broadening matrix of the left/right electrode and $G^{R / A}$ the retarded/advanced Green's function. By introducing the quantum of conductance, $G_{0}=2 e^{2} / h$, we have $G_{\alpha}\left(E=E_{F}\right)=G_{0} T_{\alpha}\left(E_{F}\right) / 2$. The total conductance amounts to $G(E)=\sum_{\alpha} G_{\alpha}(E)$. Moreover, we define the magnetoresistance as $[32,33]$

$$
\mathrm{MR}=\frac{G_{P}-G_{A P}}{G_{P}},
$$

where $\mathrm{P}$ and AP, respectively, denotes parallel and antiparallel alignment of the magnetizations of the two electrodes.

\section{RESULTS AND DISCUSSION}

Pristine graphene is nonmagnetic and shows a characteristic Dirac cone at the Fermi energy, see Fig. 2(a). Even though in the line defect the $\mathrm{C}$ coordination remains three (with bond angles between $90^{\circ}$ and $150^{\circ}$ ), the band structure is modified

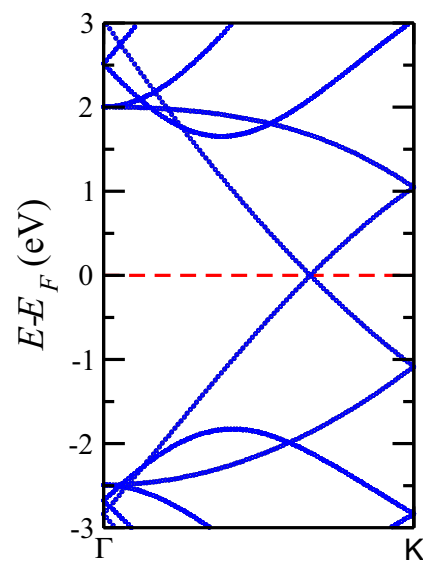

(a)

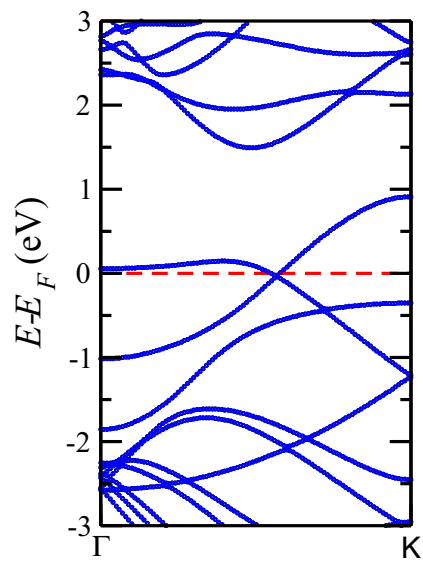

(b)
FIG. 2. (Color online) Band structures of graphene (a) without and (b) with line defect. Both cases show spin degeneracy.

prominently, see Fig. 2(b). Particularly, the system becomes metallic, while remaining nonmagnetic. The Dirac cone shows strong deformations and a slight energetic shift, so that the Dirac point appears $0.07 \mathrm{eV}$ below the Fermi energy.

The adsorption energy is given by the energy difference before and after adsorption,

$$
E=E_{\text {covered }}-E_{\text {pristine }}-E_{\mathrm{Mn}},
$$

where $E_{\text {covered }} / E_{\text {pristine }}$ are the total energies of the covered/pristine line defects and $E_{\mathrm{Mn}}$ is the total energy of the free $\mathrm{Mn}$ atoms. We find that the $\mathrm{Mn}$ atoms are most favorably absorbed on top of the C octagonal rings, confirming Ref. [31], and thus form an atomic chain along the line defect. The adsorption energy per $\mathrm{Mn}$ atom amounts to $-0.82 \mathrm{eV}$ and $-0.53 \mathrm{eV}$ for $50 \%$ and $100 \% \mathrm{Mn}$ coverage, respectively, and, according to Table I, the spin polarization of Mn is also very different (magnetic moments of $0.22 \mu_{B}$ and $0.10 \mu_{B}$ ). Both come along with significantly shorter distances of the $\mathrm{Mn}$ atoms to the graphene sheet for higher coverage. As a consequence of these deviating structural relaxation patterns, the Mn densities of states in Fig. 3 exhibit distinct differences for the two systems. In particular, the width of the $\mathrm{Mn} 4 s$ states is significantly enhanced, reflecting a strong interaction. Importantly, we observe that the $\mathrm{Mn}$ atoms induce substantial spin polarization of the electronic states at the neighboring $\mathrm{C}$ sites, see Table I. The effect is enhanced for $100 \% \mathrm{Mn}$ coverage, particularly at the Fermi energy, where we find a pronounced spin majority peak of strongly hybridized Mn and

TABLE I. Magnetic moments (in $\mu_{B}$ ) and C-Mn distances (in $\AA$ ) for the $50 \%$ and $100 \% \mathrm{Mn}$-decorated line defects.

\begin{tabular}{lccccc}
\hline \hline & \multicolumn{2}{c}{$50 \%$} & & \multicolumn{2}{c}{$100 \%$} \\
\cline { 2 - 3 } \cline { 6 - 6 } Site & Moment & Distance & & Moment & Distance \\
\hline C1 & 0.01 & 3.80 & & 0.05 & 3.32 \\
C2/C4 & 0.06 & 2.73 & & 0.13 & 2.12 \\
C3 & 0.06 & 2.82 & & 0.03 & 2.30 \\
C5 & 0.10 & 2.67 & & -0.02 & 2.34 \\
Mn & 0.22 & - & & 0.10 & - \\
\hline \hline
\end{tabular}



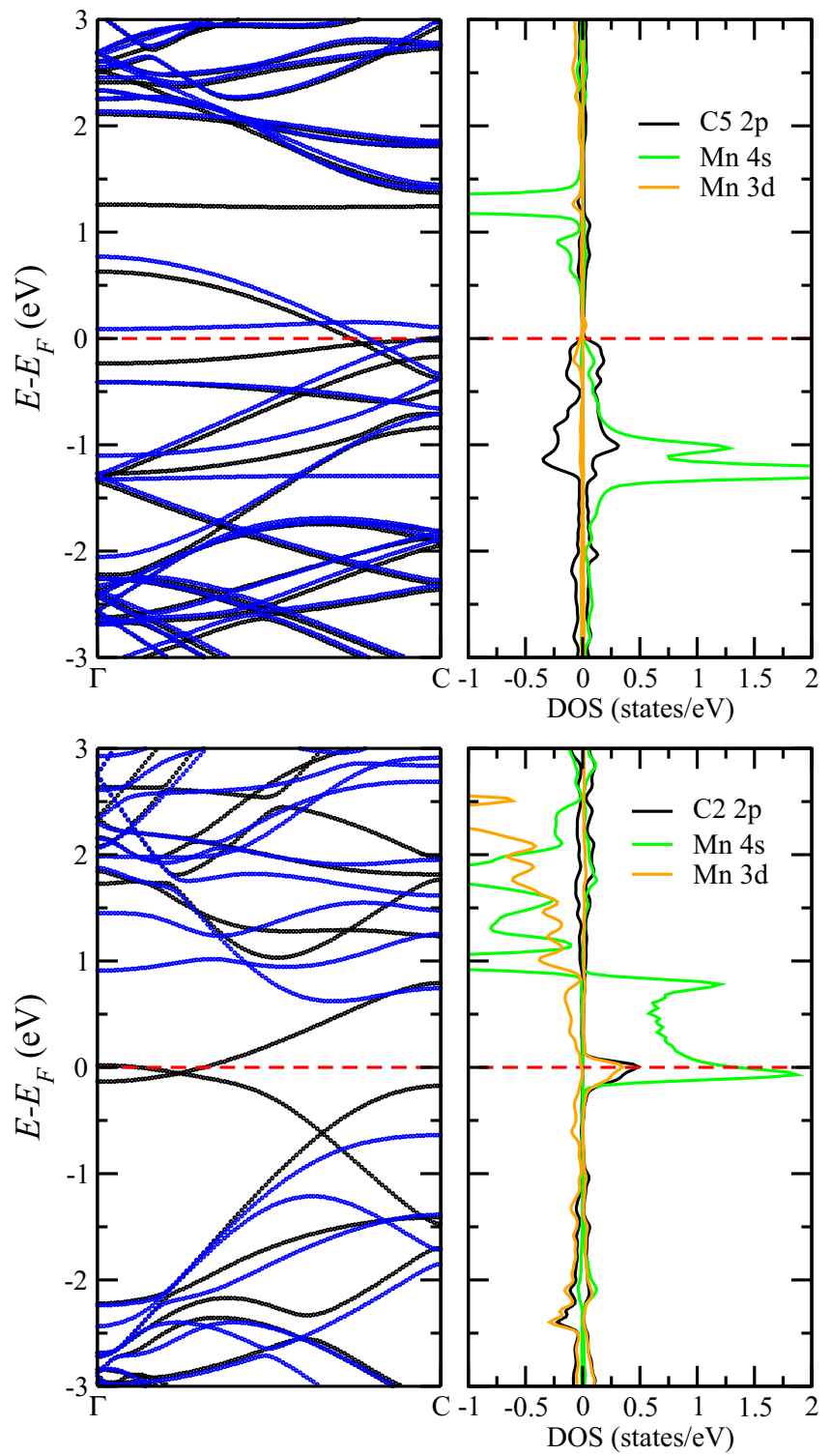

FIG. 3. (Color online) Spin polarized band structures and partial densities of states for (top) $50 \%$ and (bottom) $100 \%$ Mn coverage.

$\mathrm{C}$ states. The distance between the magnetic centers is shorter and thus the spin polarization is much less localized than in the case that each second $\mathrm{C}$ octagon does not host a Mn atom (50\% Mn coverage). The widely spread spin polarization creates a continuous path of polarized states along the line defect (C1-C2-Mn/C3-C4-C1-...) for full Mn coverage only.

The total magnetic moment $\left(4.99 \mu_{B}\right.$ and $5.07 \mu_{B}$ for $100 \%$ and $50 \%$ coverage, respectively) depends little on the Mn coverage. We also have calculated the energy difference between antiferromagnetic and ferromagnetic ordering of the Mn magnetic moments along the line defect and find the ground state to be ferromagnetic with an energy preference of $0.17 \mathrm{eV}$ per $\mathrm{Mn}$ atom. Band structures and densities of states for the Mn-decorated systems are shown in Fig. 3. The band structures indicate that for $50 \%$ Mn coverage both spin channels are metallic, while $100 \%$ Mn coverage results in a half-metallic nature. In the latter case strong hybridization

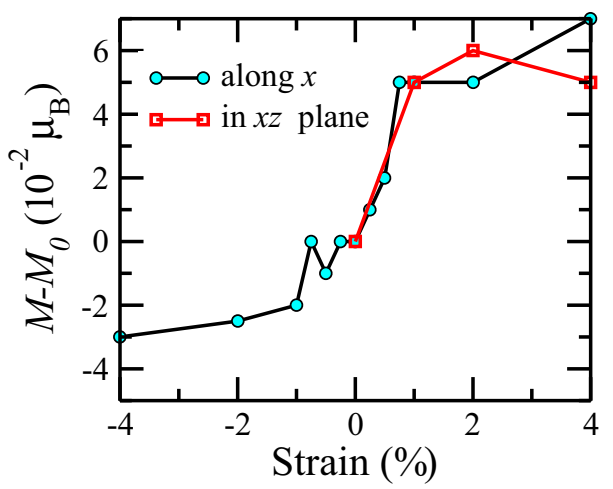

FIG. 4. (Color online) Modification of the total magnetic moment by strain along the $x$ axis and in the $x z$ plane for $100 \% \mathrm{Mn}$ coverage.

between the $\mathrm{Mn}$ and $\mathrm{C} 2$ states is visible in the density of states at the Fermi energy.

Table I summarizes the magnetic moments of the $\mathrm{Mn}$ and neighboring $\mathrm{C}$ atoms for both coverages. The rather small values obtained for $\mathrm{Mn}$ are due to strong interaction with the adjacent $\mathrm{C}$ atoms, as evidenced by the mentioned $\mathrm{Mn}-\mathrm{C}$ hybridization. Accordingly, the $\mathrm{C}$ neighbors develop a partially very significant spin polarization. We have also studied to which extent the Mn-C coupling can be modified by uniaxial or biaxial strain due to changes in the local atomic structure and therefore in the $\mathrm{Mn}-\mathrm{C}$ hybridization. The results in Fig. 4 show that the total magnetic moment increases/decreases only very slightly under tensile/compressive strain, remaining always localized on the $\mathrm{Mn}$ and neighboring $\mathrm{C}$ atoms.
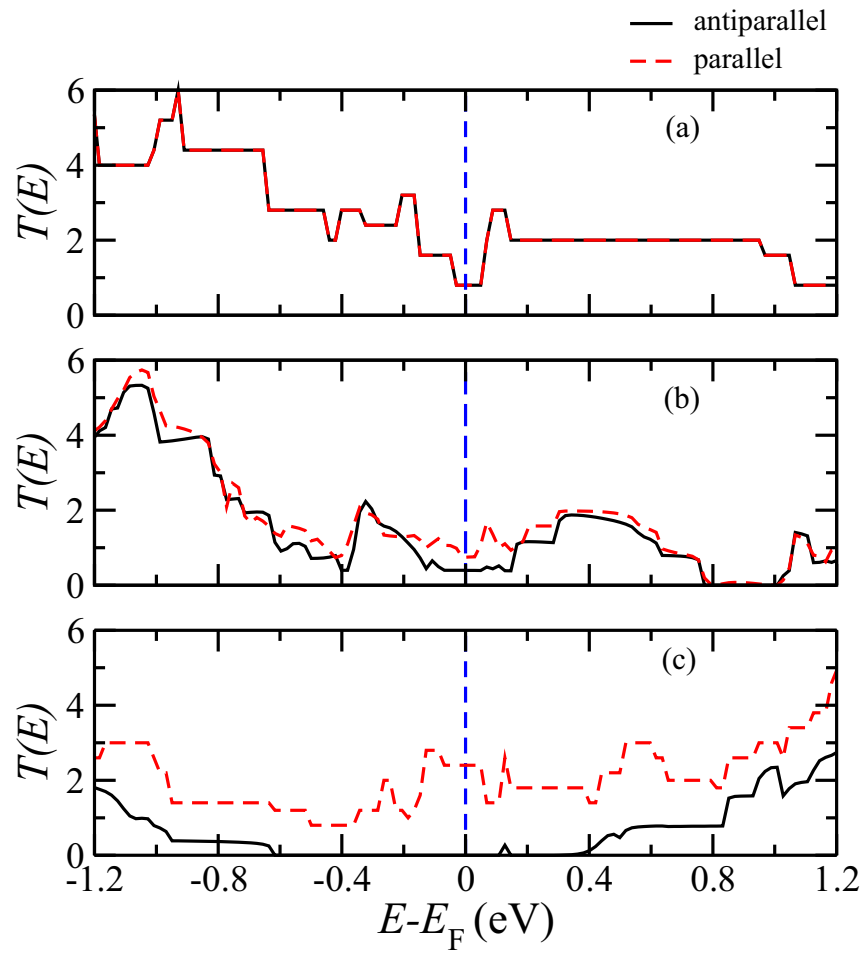

FIG. 5. (Color online) Transmission coefficient at zero bias for the (a) pristine, (b) $50 \% \mathrm{Mn}$-decorated, and (c) the $100 \% \mathrm{Mn}$ decorated line defect. 
TABLE II. Conductance and magnetoresistance at zero bias for various systems.

\begin{tabular}{lccc}
\hline \hline Line defect & $G_{P}(\mu \mathrm{S})$ & $G_{A P}(\mu \mathrm{S})$ & $\mathrm{MR}(\%)$ \\
\hline pristine & 0.62 & 0.62 & - \\
$50 \%$ Mn-decorated & 0.55 & 0.29 & 47 \\
$100 \%$ Mn-decorated & 2.40 & $10^{-11}$ & 100 \\
\hline \hline
\end{tabular}

Because of the perturbation of the $\mathrm{C}$ electronic states and the induced spin polarization, the transport properties of the line defect are strongly affected by the presence of $\mathrm{Mn}$ atoms. The zero bias transmission coefficients for the pristine [Fig. 5(a)], 50\% Mn-decorated [Fig. 5(b)], and 100\% [Fig. 5(c)] Mn-decorated line defects are shown in Fig. 5. Figure 5(a) demonstrates degeneracy between parallel (P) and antiparallel (AP) alignments of the electrode magnetizations. The transport quantization (transport channels) is clearly visible. According to Fig. 5(b), for 50\% Mn coverage both the $\mathrm{P}$ and AP configurations give rise to transmission around the Fermi energy, where the resistance is lower for the $\mathrm{P}$ configuration. This leads to a magnetoresistance of $47 \%$ at zero bias, see Table II. For 100\% Mn coverage, see Fig. 5(c), the shorter Mn-Mn distance and enhanced interaction with the $\mathrm{C}$ host (transport path of ferromagnetically coupled atoms created; see above) results in a suppression of the transmission in the AP configuration and thus a magnetoresistance of $100 \%$, compare Table II.

Turning to the dependence of the magnetoresistance on the bias voltage, Figs. 6(a) and 6(b) demonstrate for the pristine and 50\% Mn-decorated line defects essentially linear and almost identical I-V characteristics for the P and AP configurations. On the contrary, for $100 \% \mathrm{Mn}$ coverage we observe in Fig. 6(c) charging up to $0.1 \mathrm{eV}$ (due to the band gap in the minority spin channel) and thereafter a strongly suppressed $I_{A P}$, as expected from the above discussion. Under growing bias we find that $I_{A P}$ continues to grow, whereas $I_{P}$ quickly saturates. Thus, the magnetoresistance is reduced to around $70 \%$ at $V=0.4 \mathrm{~V}$.

\section{CONCLUSION}

In conclusion, we have demonstrated spin-dependent transport along a Mn-decorated topological line defect in graphene. Though it is not straightforward to realize this system, because line defects have to be created in a controlled fashion and then have to be decorated with the transition metal, the situation does not look hopeless. Pristine line defects have been observed in as-grown graphene [5] and can also be induced by electron irradiation [6,7]. In addition, substantial preferential bonding of the Mn atoms at octagonal hollow sites overcomes the problem that transition-metal atoms usually are very mobile on graphene. Interestingly, the magnetic
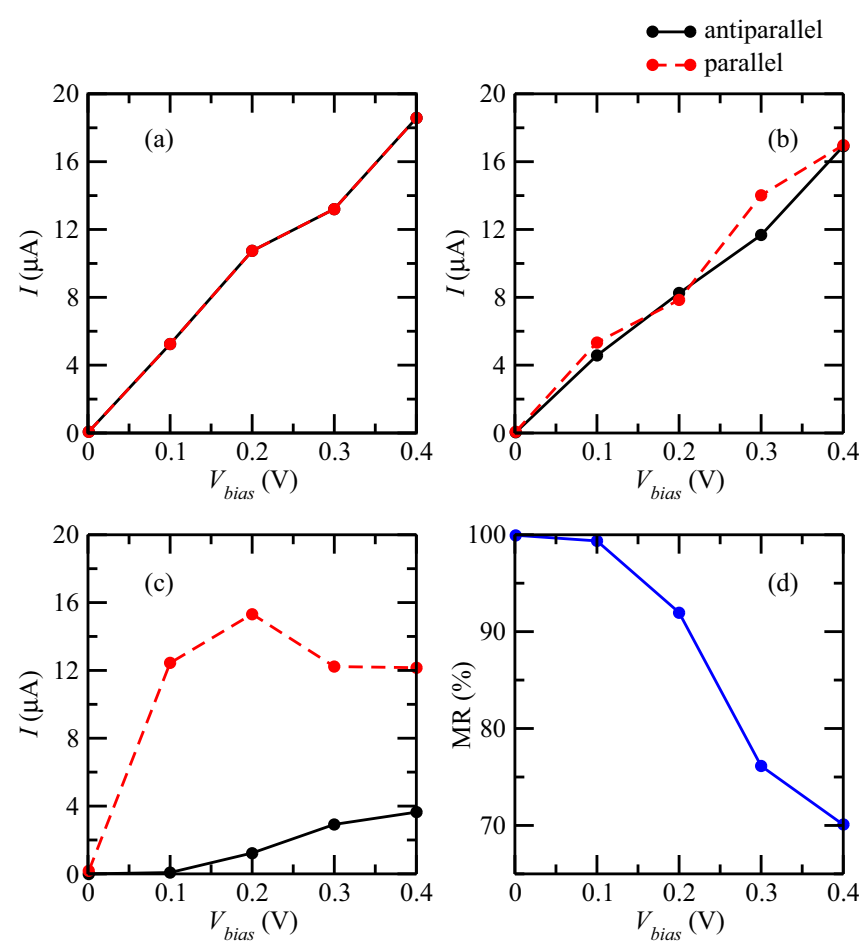

FIG. 6. (Color online) I-V characteristics for the $\mathrm{P}$ and AP configurations of the (a) pristine, (b) 50\% Mn-decorated, and (c) $100 \%$ Mn-decorated line defect. (d) Magnetoresistance for case (c).

properties depend hardly on strain, which is important as strain typically cannot be avoided in exfoliated graphene. The transport properties turn out to vary strongly with the Mn coverage. In particular, a magnetoresistance of $47 \%$ is obtained for $50 \% \mathrm{Mn}$ coverage at zero bias, which grows to $100 \%$ when the line defect is completely decorated with $\mathrm{Mn}$ atoms. For bias voltages up to $0.4 \mathrm{~V}$ high values of the magnetoresistance are maintained for the $100 \% \mathrm{Mn}$-decorated line defect, which therefore is interesting from the application point of view. The proposed system does not suffer from the limited controllability of graphene nanoribbon edges, which previously have been put forward for magnetoresistance devices. The high magnetoresistance of the Mn-decorated 8-5-5-8 topological line defect in graphene is unexpected, because the magnetic Mn impurities even for full coverage are still separated by about $5 \AA$. It turns out that the observed behavior is a consequence of induced strong $\pi$ magnetism in the graphene host.

\section{ACKNOWLEDGMENTS}

Research reported in this publication was supported by the King Abdullah University of Science and Technology (KAUST). The authors thank I. Rungger for fruitful discussions.
[1] M. S. Dresselhaus, G. Dresselhaus, and P. C. Eklund, Science of Fullerenes and Carbon Nanotubes (Academic Press, San Diego, 1996).
[2] J. W. Mintmire, B. I. Dunlap, and C. T. White, Phys. Rev. Lett. 68, 631 (1992).

[3] S. Ijima, Nature (London) 354, 56 (1991). 
[4] M. Upadhyay Kahaly, S. P. Singh, and U. V. Waghmare, Small 4, 2209 (2008).

[5] J. Lahiri, Y. Lin, P. Bozkurt, I. I. Oleynik, and M. Batzill, Nat. Nanotechnol. 5, 326 (2010).

[6] J. Kotakoski, A. V. Krasheninnikov, U. Kaiser, and J. C. Meyer, Phys. Rev. Lett. 106, 105505 (2011).

[7] J.-H. Chen, G. Autès, N. Alem, F. Gargiulo, A. Gautam, M. Linck, C. Kisielowski, O. V. Yazyev, S. G. Louie, and A. Zettl, Phys. Rev. B. 89, 121407(R) (2014).

[8] E. Cockayne, G. M. Rutter, N. P. Guisinger, J. N. Crain, P. N. First, and J. A. Stroscio, Phys. Rev. B 83, 195425 (2011).

[9] H. Terrones, R. Lv, M. Terrones, and M. S. Dresselhaus, Rep. Prog. Phys. 75, 062501 (2012).

[10] R. R. Nair, I.-L. Tsai, M. Sepioni, O. Lehtinen, J. Keinonen, A. V. Krasheninnikov, A. H. Castro Neto, M. I. Katsnelson, A. K. Geim, and I. V. Grigorieva, Nat. Commun. 4, 2010 (2013).

[11] M. Gmitra, D. Kochan, and J. Fabian, Phys. Rev. Lett. 110, 246602 (2013).

[12] M. B. Lundeberg, R. Yang, J. Renard, and J. A. Folk, Phys. Rev. Lett. 110, 156601 (2013)

[13] Z. Tehrani, G. Burwell, M. A. M. Azmi, A. Castaing, R. Rickman, J. Almarashi, P. Dunstan, A. M. Beigi, S. H. Doak, and O. J. Guy, 2D Mater. 1, 025004 (2014).

[14] R. Yang, S. Wu, D. Wang, G. Xie, M. Cheng, G. Wang, W. Yang, P. Chen, D. Shi, and G. Zhang, Nano Res. 7, 1449 (2014).

[15] G. Kim, S.-H. Jhi, S. Lim, and N. Park, Appl. Phys. Lett. 94, 173102 (2009).

[16] J. A. Rodrìguez-Manzo, O. Cretu, and F. Banhart, ACS Nano 4, 3422 (2010).

[17] A. V. Krasheninnikov, P. O. Lehtinen, A. S. Foster, P. Pyykkö, and R. M. Nieminen, Phys. Rev. Lett. 102, 126807 (2009).
[18] O. Cretu, A. V. Krasheninnikov, J. A. Rodrìguez-Manzo, L. Sun, R. M. Nieminen, and F. Banhart, Phys. Rev. Lett. 105, 196102 (2010).

[19] Y. Tang, Z. Yang, and X. Dai, J. Chem. Phys. 135, 224704 (2011).

[20] K. Kim, H.-B.-R. Lee, R. W. Johnson, J. T. Tanskanen, N. Liu, M.-G. Kim, C. Pang, C. Ahn, S. F. Bent, and Z. Bao, Nat. Commun. 5, 4781 (2014).

[21] K. M. H. Lenssen, D. J. Adelerhof, H. J. Gassen, A. E. T. Kuiper, G. H. J. Somers, and J. B. A. D. van Zon, Sens. and Actuators A 85, 1 (2000).

[22] M. Pannetier-Lecoeur, C. Fermon, A. de Vismes, E. Kerr, and L. Vieux-Rochaz, J. Magn. Magn. Mater. 316, e246 (2007).

[23] B. Hu and Y. Wu, Nat. Mater. 6, 985 (2007)

[24] J. Bai, R. Cheng, F. Xiu, L. Liao, M. Wang, A. Shailos, K. L. Wang, Y. Huang, and X. Duan, Nat. Nanotechnol. 5, 655 (2010).

[25] M. Y. Han, J. C. Brant, and P. Kim, Phys. Rev. Lett. 104, 056801 (2010).

[26] W. Li, M. Zhao, Y. Xia, R. Zhang, and Y. Mu, J. Mater. Chem. 19, 9274 (2009).

[27] N. A. Pike and D. Stroud, Appl. Phys. Lett. 105, 052404 (2014).

[28] E. E. Mallon, M. Y. Jeon, M. Navarro, A. Bhan, and M. Tsapatsis, Langmuir 29, 6546 (2013).

[29] P. Ordejon, E. Artacho, and J. M. Soler, Phys. Rev. B 53, R10441 (1996).

[30] A. R. Rocha, V. M. Garcia-Suarez, S. W. Bailey, C. J. Lambert, J. Ferrer, and S. Sanvito, Nat. Mater. 4, 335 (2005).

[31] Z. Zhu, W. Chen, Q. Sun, and Y. Jia, J. Phys. D: Appl. Phys. 47, 055303 (2014).

[32] J. M. de Almeida, A. R. Rocha, A. J. R. da Silva, and A. Fazzio, Phys. Rev. B 84, 085412 (2011).

[33] V. M. Karpan, P. A. Khomyakov, A. A. Starikov, G. Giovannetti, M. Zwierzycki, M. Talanana, G. Brocks, J. van den Brink, and P. J. Kelly, Phys. Rev. B 78, 195419 (2008). 